\title{
Simulating observable comets
}

\section{The effects of a single stellar passage through or near the Oort cometary cloud}

\author{
P. A. Dybczyński ${ }^{\star}$ \\ Astronomical Observatory of the A. Mickiewicz University, Słoneczna 36, 60-286 Poznań, Poland \\ Received 2 July 2002 / Accepted 9 September 2002

\begin{abstract}
This is the first of a series of papers presenting an attempt to reproduce the mechanisms acting currently on the Oort cloud of comets and producing the observed long-period comets. In this first part we give a short review of papers on the stellar perturbations on the cometary cloud and on the observed long-period comet perihelia/aphelia directions distribution. We present in detail the effects of a single stellar passage through or near the cometary cloud by means of a Monte Carlo simulation based on the improved impulse approximation. The simulations are performed for two different steady-state numerical models of the Oort cloud and for different parameters of the stellar passage. Among others, we calculate the probabilities of producing an observable comet as well as ejecting it from the cloud, we characterize cometary shower resulting from the close stellar passage and discuss anisotropies in the observable sample of comets, produced by a simulated single stellar perturbation.
\end{abstract}

Key words. comets: general - Oort cloud - solar system: general

\section{Introduction}

When presenting his concept of the cometary cloud surrounding the Solar System, Oort (1950) proposed stellar perturbations as the only source of perturbations that changes cometary orbits and makes them observable. Since that time we have recognized that there is another important source of perturbations in the cometary cloud: the galaxy as a whole or, to a first approximation, the galactic disk. However, as we show below, stellar perturbations play a key role in delivering comets into the planetary region. Both stellar and galactic perturbations act simultaneously on the cometary cloud and the aim of this series of papers is to reproduce their "net effect" by means of a computer Monte Carlo simulation. It should be noted that we are interested here only in last 10-20 million years of the Oort cloud history, so such potentially significant events as encounters with giant molecular clouds are omitted in our calculations. Planetary perturbations are also completely ignored.

Many authors (e.g. Heisler \& Tremaine 1986; Bailey et al. 1990) have stated that the galactic field tidal effect is more efficient than stellar perturbations in producing observable comets but these two agents work in a completely different manner. Galactic tide is a permanent perturbation that causes periodic, long-term variation of cometary orbital elements, including perihelion distance. It may be shown (see for example: Dybczyński \& Prȩtka 1996; Breiter et al. 1996;

\footnotetext{
^ email: dybol@amu.edu.pl
}

Maciejewski \& Prętka 1998) that only a small part (approximately one tenth) of the whole Oort cloud can become observable due to the disk tide in the absence of other perturbing forces. Also, comets cannot migrate between potentially observable and unobservable parts of the Oort cloud population. This means that without other types of perturbation (mainly stellar) that can randomize the whole population, all potentially observable comets would have been completely removed by planetary perturbations and/or physical decay a long time ago, taking into account the age of the Solar System. This is the first reason why we want to investigate stellar perturbations in detail before starting to investigate simultaneous stellar and galactic disk tide action, which we describe in the second paper of this series.

We are also interested in looking for asymmetries in the distribution of perihelia/aphelia directions of the observed longperiod comets. There exist a large number of papers on this subject, see for example: Carrington (1861), Tyror (1957), Hurnik (1964), Hasegawa (1976), Oja (1975), Yabushita (1979b,a), Bogart \& Noerdlinger (1982), Khana \& Sharma (1983), Biermann et al. (1983), Lüst (1984), Brunini (1993), Matese et al. (1998); Matese et al. (1999). Apart from some depletions near the galactic equator and in pole regions caused by galactic tide (discussed among others by Delsemme 1987), stellar passage seems to be a good candidate for a mechanism producing some kind of grouping of perihelia directions in an arbitrary place on the celestial sphere. We show several examples of such groupings for different parameters of such a passage. 


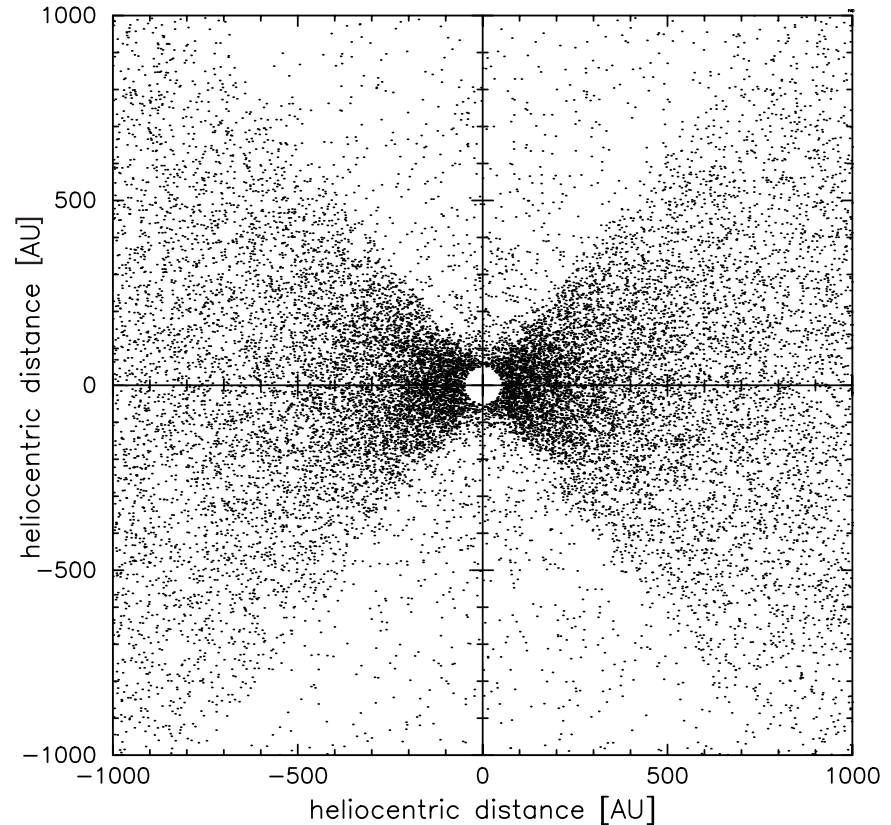

Fig. 1. Instantaneous positions of simulated comets in the intersection (perpendicular to the invariable plane) of the innermost $1000 \mathrm{AU}$ of the DLDW model of the Oort cloud. The distribution of the cometary bodies is highly concentrated towards the invariable plane of the Solar System.

Finally, the third reason for the importance of the stellar perturbations on the Oort cloud is the problem of the so called "cometary showers", i.e. the temporal, significant increase of the observable comet influx after a close (or penetrating) stellar passage. This concept was first introduced by Hills (1981) and then discussed in many papers (see for example: Weissman 1984; Weissman \& Hut 1986; Fernández \& Ip 1987; Heisler et al. 1987; Hut et al. 1987; Heisler et al. 1991; Matese et al. 1998). From the point of view of cometary origin theories it is very important to judge whether we are now observing comets during a cometary shower or the observed flux is at the background level. To answer this question it is necessary first to describe in detail the structure and time-dependence of the cometary shower induced by the single stellar passage and then to search for candidate stars among those which traversed the Solar System lately. The third paper of this series will describe available datasets, the search strategy and the results.

In the second paper of this series we also discuss the recently published paper by Matese \& Lissauer (2002) and in the third part the recent paper by García-Sánchez et al. (2001).

\section{Two models of the current Oort cloud}

To develop the Monte Carlo simulation computer code for the recent stellar passage through or near the cometary cloud, one has to describe the steady-state distribution of cometary orbits in the cloud. In this paper we use two distinct models of the current, steady-state Oort cloud. The first one, which may be called classical, is based on the results of the Duncan et al. (1987) simulation of the cloud formation; we call this model DQT. These distributions were first used in the Monte Carlo simulations of

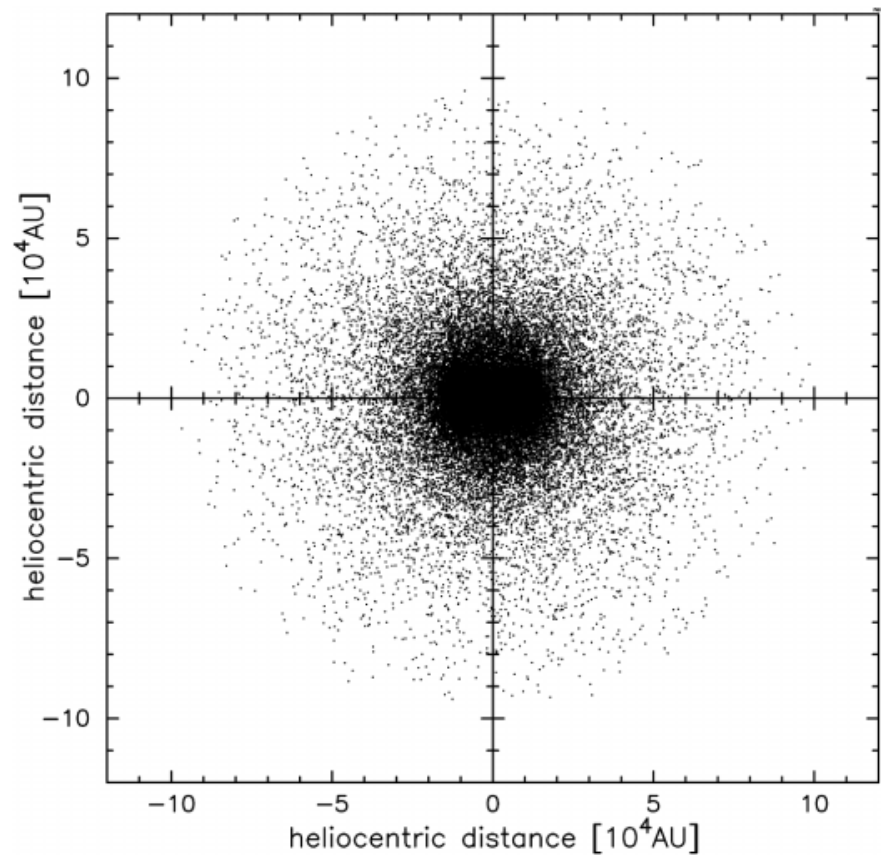

Fig. 2. The overall view of the DLDW model of the Oort cometary cloud. One can notice that the outer part of the cloud is almost spherically symmetric. This picture may be compared with Fig. 1 of Weissman (1996), obtained for the DQT model.

star passages by Weissman (1996). To obtain the random orbit generator for this model we numerically reconstructed the probability density of the semimajor axis of cometary orbits from Fig. 8 of Duncan et al. (1987) and used the eccentricity probability density function, proposed by Hills (1981):

$f(e)=2 e$

which is consistent with numerical results obtained by Duncan et al. (1987). Semimajor axes and eccentricities were treated here as independent random variables. The rest of the orbital elements were generated in such that they define the spherically symmetric cometary cloud. An example of the cometary cloud simulated according to this model may be found in Fig. 1 of Weissman (1996).

The second model is based on the recent simulations of the Oort cloud formation by Dones et al. (2000b, 2000a). Hereafter we call it DLDW. Based on their results we numerically reconstructed the semimajor axis, eccentricity and inclination distributions. In this model it was necessary to take into account the dependence of the eccentricity and the inclination on the semimajor axis of a comet because such dependences are evident in plots obtained by Dones et al. (2000a). The resulting cometary cloud is significantly different from that of the DQT model: the majority of comets is situated in the outer part and the inner part is not spherically symmetric but flattened.

In Fig. 1 we present the innermost part of the simulated Oort cloud, according to the DLDW model. While this inner part is significantly flattened, the outer part of the cometary cloud is almost spherically symmetric (see Fig. 2). The comparison of the cometary body densities with respect to the heliocentric distance for the two models described above is presented in Fig. 3. Both curves describe the cloud of the same 


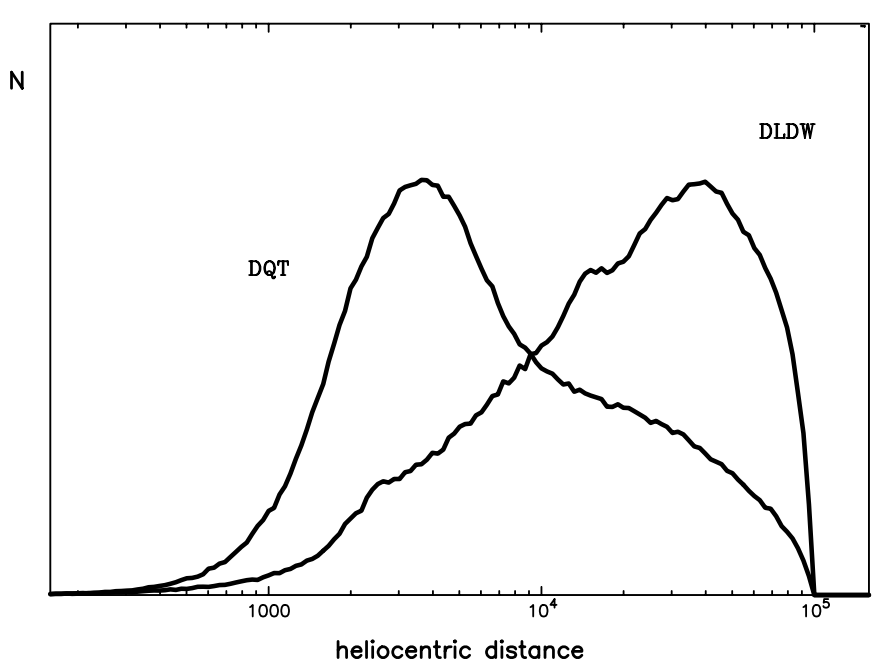

Fig. 3. The number of cometary bodies at different heliocentric distances obtained from the Monte Carlo simulations for two different models of the Oort cloud.

number of comets and are limited according to the inner and outer boundaries of the cloud adopted in this paper. The outer boundary is set to be equal to $1 \times 10^{5} \mathrm{AU}$, which is the typical value. From the qualitative analysis of Dones et al. (2000a) it appears that the Oort cloud starts at a semimajor axis equal to about $1000 \mathrm{AU}$. Our application of their model is therefore limited in the semimajor axis to the interval from $1 \times 10^{3}$ to $1 \times 10^{5} \mathrm{AU}$. In this paper we choose $50 \mathrm{AU}$ as the inner boundary for a simulated comet position.

These models may be treated as the two opposite extreme cases from the point of view of the inner to outer ratio, as is illustrated in Fig. 3. In the DQT model the inner part of the cloud dominates while in the DLDW model the outer part is much more populated and the inner part almost absent. In this paper we present the detailed results of the stellar passage for both models which makes it applicable to the wide range of ratio value mentioned above. According to Dones (2002, private communication) the main reason for the remarkable differences between the two models presented is that DQT started with highly eccentric orbits, which prevented the comet perihelia from evolving due to planetary perturbations. Thus Neptune and Uranus could inject comets directly into the Oort Cloud. By contrast, in DLDW they started with nearly circular orbits. Many Uranus-Neptune objects evolve inward to be placed into the Oort Cloud by Saturn and Jupiter, which throw them further out because their greater mass and smaller semi-major axis provides much larger energy perturbations. The net result is that DQT's Oort cloud is much more centrally concentrated than in DLDW.

\section{The separate stellar action}

Stellar perturbations of the cometary orbits in the Oort cloud may be calculated by the so-called impulse approximation, first proposed by Öpik (1932) and then successfully used by Oort in his paper introducing the concept of a cometary cloud surrounding the Sun (Oort 1950). This method is the approximation of the three body problem (Sun-star-comet) based on the fact that during the close stellar passage a comet can be treated as motionless with respect to the Sun. Such a stellar passage lasts typically several thousand years only while the comet moves very slowly, most often near its aphelion, tens of thousands of AU from the Sun. In this approximation we take into account the complete effect of the stellar perturbation by applying some velocity impulse to the comet at the instant of the closest stellar passage. In its classical form, the impulse approximation is based additionally on the assumption of the straight line motion of the star so that it ignores the influence of the Sun on a star trajectory. The total effect of the stellar passage is expressed as a cometary velocity change proportional to the mass of the star $M_{\star}$ and inversely proportional to the product of the minimum star-comet distance and the stellar velocity. The final change in the comet heliocentric orbit is calculated using the difference between the velocity impulses gained by the Sun and the comet itself. The complete derivation of the classical version of the impulse approximation as well as the detailed discussion of its assumptions may be found in Rickman (1976). The classical impulse approximation was used by many authors, see for example: Fernández (1980); Weissman (1980); Scholl et al. (1982); Yabushita et al. (1982); Remy \& Mignard (1985); Rickman \& Froeschle (1988); García-Sánchez et al. (1999).

However, it is possible to take into account the Sun-star gravitational attraction by embedding the two-body solution for heliocentric stellar motion in modified velocity impulse formulae. We call this method the improved impulse approximation and we use it in all our simulations. It was first proposed by Dybczyński (1994). It fully accounts for the Sun-star interaction and the resulting curvature of the stellar trajectory when calculating direction and strength of velocity impulses gained by both the comet and the Sun. The improved impulse approximation has been shown to be superior to the classical version in the case of a small comet-star distance during a stellar passage. In a Monte Carlo simulation of such a passage through the Oort cloud, this distance can be arbitrarily small. A comparison with the numerical integration of the Sun-comet-star motion showed that the improved impulse approximation gives a very good estimate of the stellar perturbations in comet motion in a very efficient way. It works 500 times faster than the numerical integration. This method was successfully applied in our previous Monte Carlo simulations (Dybczyński 1990) to study the probability of producing observable comets for the simplified model of the cloud.

A set of formulae for calculating the complete effect of the stellar passage on cometary orbit was obtained by Dybczyński (1994). For simplicity, these formulas are derived in the reference frame aligned with the heliocentric orbit of the star (hereafter we call it the "stellar" frame, see the quoted paper for details). To allow for combining stellar and galactic perturbations in future calculations and to maintain the compatibility between all three papers of this series we define here the stellar path as an ordinary heliocentric, hyperbolic orbit. It is therefore defined by the perihelion distance $q_{\star}$, the star velocity at infinity $V_{\infty}$ and the orientation of the stellar orbit is described with ordinary angular elements, measured with respect to the galactic disk plane. The eccentricity of the heliocentric stellar 


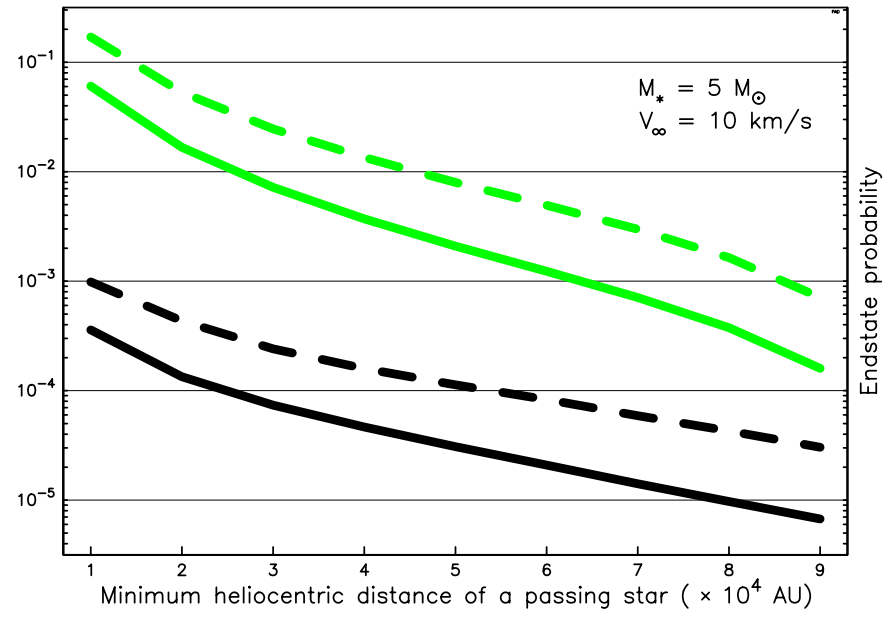

Fig. 4. The dependence of the probability of being lost (in gray) or becoming observable (in black) on the minimal Sun-star distance for two different Oort cloud models. Solid lines describe results for the DQT model while dashed lines correspond to the DLDW model.

orbit defined in this manner is given by ( $k$ is the Gaussian gravitational constant):

$e_{\star}=\frac{q_{\star} V_{\infty}^{2}}{k^{2}\left(1+M_{\star}\right)}+1$

and typically it is significantly greater than one: for $q_{\star}=30000 \mathrm{AU}, V_{\infty}=20 \mathrm{~km} \mathrm{~s}^{-1}$ and $M_{*}=1 M_{\odot}$ we obtain $e_{\star}=6755.336$ !

In this paper we also analyze the influence of the stellar path orientation on the resulting observable cometary sample. The obvious reason for this emerges from combining the stellar and galactic perturbations, which will be discussed in the second paper of this series. However, because the DLDW model of the Oort cloud is not spherically symmetric, taking into account the orientation of the star heliocentric orbit is necessary even in the absence of the galactic perturbations in the simulation. The innermost part of DLDW model of the cloud is significantly flattened towards the invariable plane of the Solar System (see Fig. 1) so it is interesting to search for any asymmetries in the observable comet sample resulting from this flattened distribution.

The formulae for calculating the cometary velocity impulse in rectangular components in the "stellar" reference frame are:

$$
\begin{aligned}
\Delta v_{x} & =-\frac{2 k^{2} M_{\star}}{V_{\infty}}\left(\frac{a_{\mathrm{c}}}{c_{\mathrm{c}}^{2}}-\frac{a_{\mathrm{s}}}{c_{\mathrm{s}}^{2}}\right), \\
\Delta v_{y} & =\frac{2 k^{2} M_{\star}}{V_{\infty}}\left(\frac{b_{\mathrm{s}}-y_{\mathrm{c}}}{c_{\mathrm{c}}^{2}}-\frac{a_{\mathrm{s}}}{c_{\mathrm{s}}^{2}}\right), \\
\Delta v_{z} & =-\frac{2 k^{2} M_{\star}}{V_{\infty}}\left(\frac{z_{\mathrm{c}}}{c_{\mathrm{c}}^{2}}\right),
\end{aligned}
$$

where $\Delta v_{x}, \Delta v_{y}, \Delta v_{z}$ are heliocentric velocity impulse components while

$a_{\mathrm{c}}=k^{2} M_{*} / V_{\infty}^{2}$

$a_{\mathrm{s}}=k^{2}\left(M_{*}+1\right) / V_{\infty}^{2}$

$b_{\mathrm{s}}=a_{\mathrm{s}} \sqrt{e_{\star}^{2}-1}$

$c_{\mathrm{s}}^{2}=a_{\mathrm{s}}^{2}+b_{\mathrm{s}}^{2}$

$c_{\mathrm{c}}^{2}=a_{\mathrm{c}}^{2}+\left(b_{\mathrm{s}}-y_{\mathrm{c}}\right)^{2}+z_{\mathrm{c}}^{2}$

and $x_{\mathrm{c}}, y_{\mathrm{c}}, z_{\mathrm{c}}$ are coordinates of a comet in the adopted reference frame. The detailed derivation of the above formulae one can find in Dybczyński (1994).

\section{The Monte Carlo simulation scheme}

There are several papers on simulating the stellar passages near or through the cometary cloud. Early Weissman simulations (Weissman 1977, 1979, 1982, 1984) were highly simplified by representing stellar perturbations with velocity impulses random in direction, proportional to the cometary orbital period and applied only at the comet's aphelion. They are also performed for non-realistic initial distributions of cometary orbits in the cloud, see Remy \& Mignard (1985) for a more detailed review. Fernández $(1980,1982)$ used a more advanced model, introducing the stellar path geometry and accounting for the differential nature of the perturbation, but his simulations of the cometary cloud evolution concentrated mainly on the formation of the Oort cloud under the influence of the planetary and stellar perturbations during the early stage of the Solar System, so they were performed for the flat initial inclination distribution of proto-comets, located in the Uranus-Neptune zone. In this simulation, the number of comets in the sample varied from 1000 to 3000 . These simulations were performed for long time intervals, therefore he accounted for many different, successive stellar passages and did not present any detail on the results of a single close encounter. Remy \& Mignard (1985) used even smaller samples (typically 200 comets) with different initial distributions of perihelion and aphelion distances for the spherically symmetric cloud.

All the simulations mentioned so far ignored galactic disk tide.Two papers by Julia Heisler $(1987 ; 1990)$ describe simultaneous galactic and stellar perturbations on the Oort cloud, modeled by means of the Monte Carlo simulations. They were performed for the time intervals comparable with the age of the Solar System and allowed for the multiple, consecutive random stellar passages so anisotropies due to the stellar path geometries could not be obtained. Later on, Heisler et al. (1991) attempted to describe the sky distribution of the Oort cloud comets during the "shower" basing on the simplified cloud model. Finally, Weissman (1996) concluded that even close stellar passage produces an isotropic observable comet population. We show in Sect. 6 that this conclusion is false (as was shown recently by Dybczyński 2002a,b).

In this paper we concentrate on the results of the single stellar passage taking into account its geometry, analyzing spatial distributions and time dependence of the resulting observable sample of comets. 


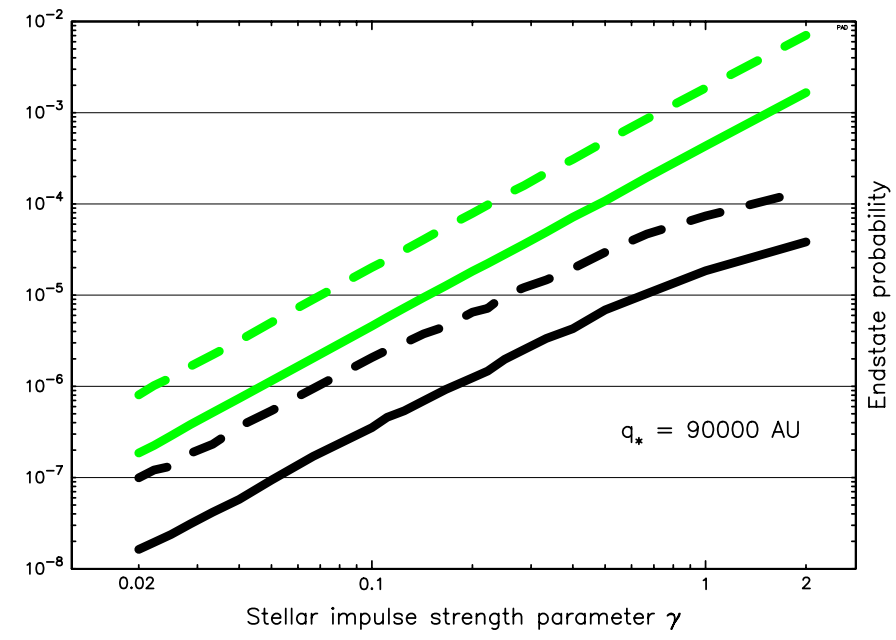

Fig. 5. The dependence of the probability of being lost (in gray) and becomig observable (in black) on the stellar impulse strength for two different cloud models and the proximity distance equal to $90000 \mathrm{AU}$. Dashed lines mark results for the DLDW model while solid lines correspond to the DQT one.

Using the impulse approximation one can calculate the effects of a single stellar passage through or near the Oort cometary cloud by means of the Monte Carlo simulation. After choosing the parameters of the stellar encounter $\left(M_{\star}, q_{\star}, V_{\infty}\right.$, $\Omega_{\star}, \omega_{\star}, i_{\star}$ defined in the galactic frame) and deciding which model of the cometary cloud to be used, we can work with the following scheme:

1. Randomly choose orbital elements of a comet according to the cometary cloud model adopted (the orientation of the orbit is defined here with respect to the invariable plane of the Solar System to allow for flattened clouds),

2. Choose the mean anomaly of a comet (uniformly distributed between 0 and $2 \pi$ ),

3. Calculate the heliocentric position and velocity of a comet and change the reference plane from the invariable plane through the galactic disk plane to the heliocentric stellar orbit plane,

4. Apply the improved impulse approximation formulas modifying cometary velocity and then transform the position and velocity of a comet back to the galactic frame for new orbit calculation,

5. Calculate and examine the new heliocentric cometary orbit (one can count observable or ejected comets, accumulate perihelion direction distribution for observable comets etc.)

6. Repeat steps $1-5$ until a statistically significant sample of orbits is accumulated.

On a contemporary desktop personal computer it is possible to test a sample of comets from the cloud as large as $10^{11}$ bodies using several weeks of CPU time.

In all our simulations we treat as observable all comets with perihelion distance reduced below $5 \mathrm{AU}$. We developed the original, multipurpose computer code to perform such simulations for different star passage parameters and different cloud models. This code is used to obtain end-state probabilities as

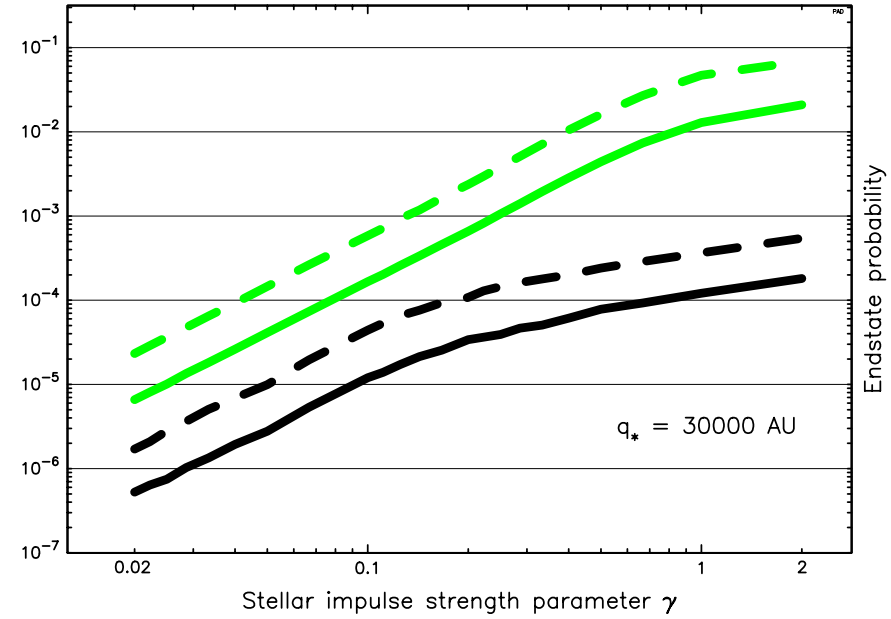

Fig. 6. The dependence of the probability of being lost (in gray) and becomig observable (in black) on the stellar impulse strength for two different cloud models and the proximity distance equal to $30000 \mathrm{AU}$. Dashed lines mark results for the DLDW model while solid lines correspond to the DQT one.

well as perihelion direction distribution and cometary shower time dependence and geometry.

\section{End states probabilities}

In this paper we consider two interesting end-states of the cometary orbit evolution under the stellar perturbation:

1. We call a comet as observable when its perihelion distance is reduced below the observability threshold, assumed here to be equal $5 \mathrm{AU}$.

2. We call a comet lost if its eccentricity become greater or equal to unity or its aphelion distance become greater than the "stellar lost limit", assumed to be equal $2.5 \times 10^{5} \mathrm{AU}$.

In both cases a comet can no longer be called a member of the Oort cloud. We allow for a comet to be counted both as observable and lost, because it is possible that before being lost a comet can pass near the Sun, closer than the observability limit. The number of such comets is marginal however, in agreement with the fact of the observing a small number of hyperbolic comets. Apart of those two end-states, there exist a third possibility: a comet remains as a member of the Oort cloud but with slightly modified orbit. In this investigation we are especially interested in the "observable" end-state because we attempted to look for fingerprints of the recent stellar perturbation in the observed long-period comet sample (this will be described in the third paper of this series).

First we present the example of the endstate probability dependence on the stellar proximity distance. In Fig. 4 we present the compilation of results of several Monte Carlo simulations for rather massive and slowly moving star to increase the strength of the stellar impulse, namely all these simulations were performed for a star of $M_{\star}=5 M_{\odot}$, moving in a hyperbolic orbit with $V_{\infty}=10 \mathrm{~km} \mathrm{~s}^{-1}$. The tested cometary cloud population varied from $1 \times 10^{7}$ to $1.5 \times 10^{9}$ bodies depending on the minimal Sun-star distance and the model. Due to this 


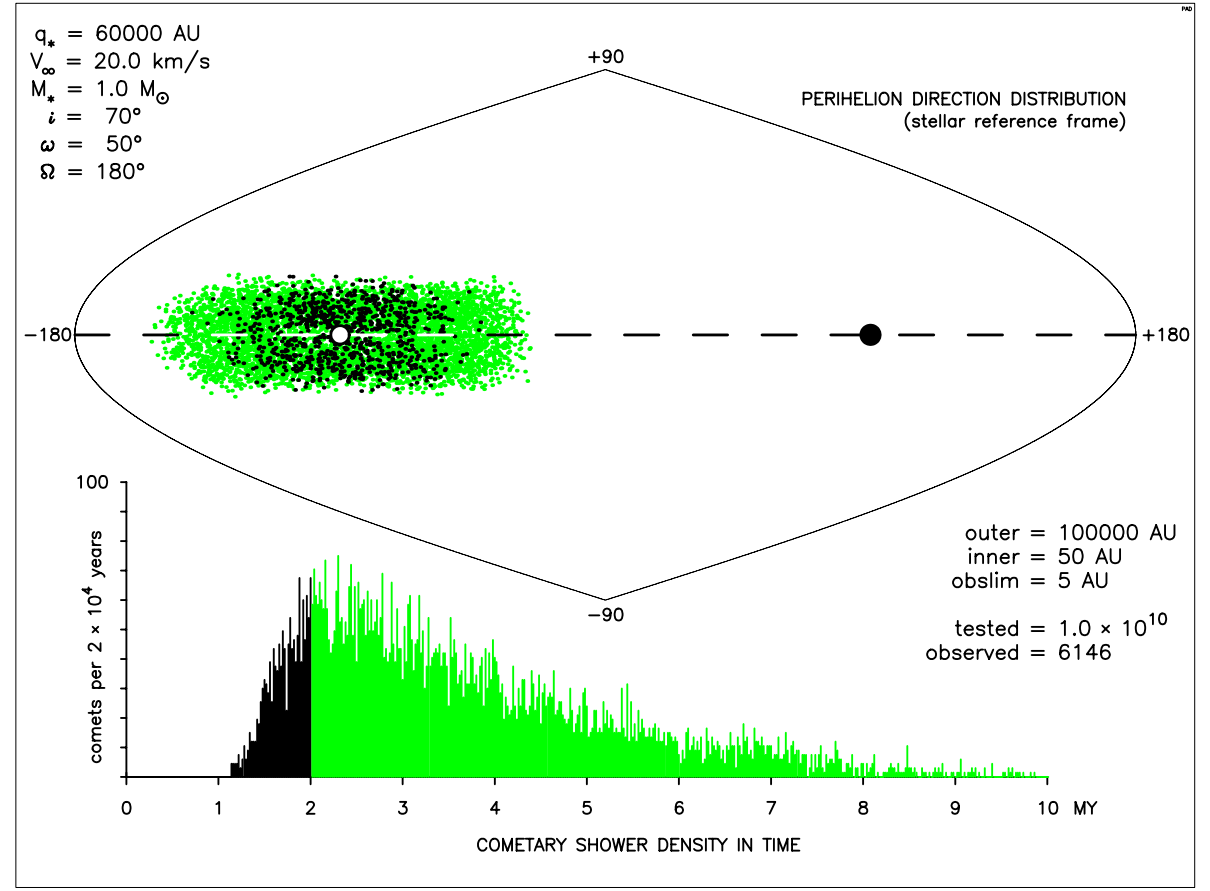

Fig. 7. The results of the simulation for the modest $q_{\star}=60000 \mathrm{AU}$. The stellar reference frame is used here so the star heliocentric orbit plane projection is represented by the dashed straight line. The star perihelion is marked with a full circle while the antiperihelion with the open one. All perihelion directions are highly concentrated around the star anti-perihelion direction on the celestial sphere. Comets arriving in the solar vicinity during the first $2 \mathrm{Myr}$ after the stellar passage are marked in black while the rest is plotted in gray. In the lower right part of this picture the main parameters of the cloud are presented. Among others "obslim" means the observability threshold used. large number of tested comets the probability error bars are all well below the scale of this picture.

One can easily observe at first the strong dependence on the cometary cloud model. Presented probabilities for the DLDW model (dashed lines in Fig. 4) are 3-4 times larger than for the DQT model (solid lines) in all corresponding cases. It means that if we estimate the present population of the Oort cloud comets basing on the observed long-period cometary flux the result will be significantly model-dependent. From the other hand, if we could estimate this number in some other way (e.g. basing on Solar System origin simulations) the observed flux might serve as a constraint for the present state cloud modeling.

In Figs. 5 and 6 we present the probability of being observed (in black) or lost (in gray) as a function of the stellar impulse strength. We present here the plots for the two cometary cloud models. Solid lines denote results for the DQT model and dashed ones for DLDW. We compared here the results for two different stellar perihelion distances $q_{\star}=30000 \mathrm{AU}$ and $q_{\star}=90000$ AU. As shown in many previous papers, it is not necessary to investigate these probabilities for all combinations of $M_{\star}$ and $V_{\infty}$ values because the coefficient:

$\gamma=\frac{M_{\star}}{V_{\infty}}$

is simply the impulse strength-measuring coefficient. If we consider stars of masses from 1 to 10 solar masses, moving at velocities from 5 to $50 \mathrm{~km} \mathrm{~s}^{-1}$ we obtain $0.02 \leq \gamma \leq 2.0$.

As expected, all these probabilities are almost linearly dependent on $\gamma$, except for the strongest impulses, where some departures from the linear dependency are observed. In all cases the DLDW model gives probabilities approximately four times greater than for the DQT model. It should be stressed here that each curve presented in Figs. 5 and 6 is based on 30 independent Monte Carlo simulations for cloud populations from $9.3 \times 10^{6}$ to $6.2 \times 10^{10}$ comets, depending on $\gamma$ and the cloud model used.

\section{Observable sample asymmetries}

The investigations of asymmetries in the distribution of the line of apsides orientation of the observed sample of long-period comets published so far concentrated on two completely different groups.

First, when discussing the cometary origin hypotheses (stellar versus solar) many attempts were made to find a correlation between some concentrations of the perihelion point directions on the celestial sphere and the solar apex. Among earliest papers in this line of research we note that by Carrington (1861). The possible effect of the solar motion in the distribution of perihelions of long-period comets was then extensively discussed by Hurnik (1964), Oja (1975), Hasegawa (1976), Bogart \& Noerdlinger (1982) and Khana \& Sharma (1983).

The second group of papers deals with the effect of asymmetric perturbing agents acting on the originally spherically symmetric population of comets. Joss (1973) discussed the cumulative effect of planetary perturbations. Biermann et al. (1983) studied the statistical evidence for the cometary aphelia grouping and proposed stellar passages as the source of the observed anisotropies in the carefully selected sample of long-period comets with well-determined orbits. Lüst (1984) extended the search for these anisotropies, studying the population of 223 long-period comets and examining the aphelia distributions in galactic, equatorial and "solar apex" coordinate systems. In a series of papers Matese (Matese \& Whitman 1989, 1992; Matese \& Whitmire 1996; Matese et al. 1998) demonstrated that some asymmetries are direct results of the galactic tidal disk force acting on comets in the Oort cloud in accordance with previous suggestions by Delsemme (1987). It was shown that galactic perturbations caused the apside line 


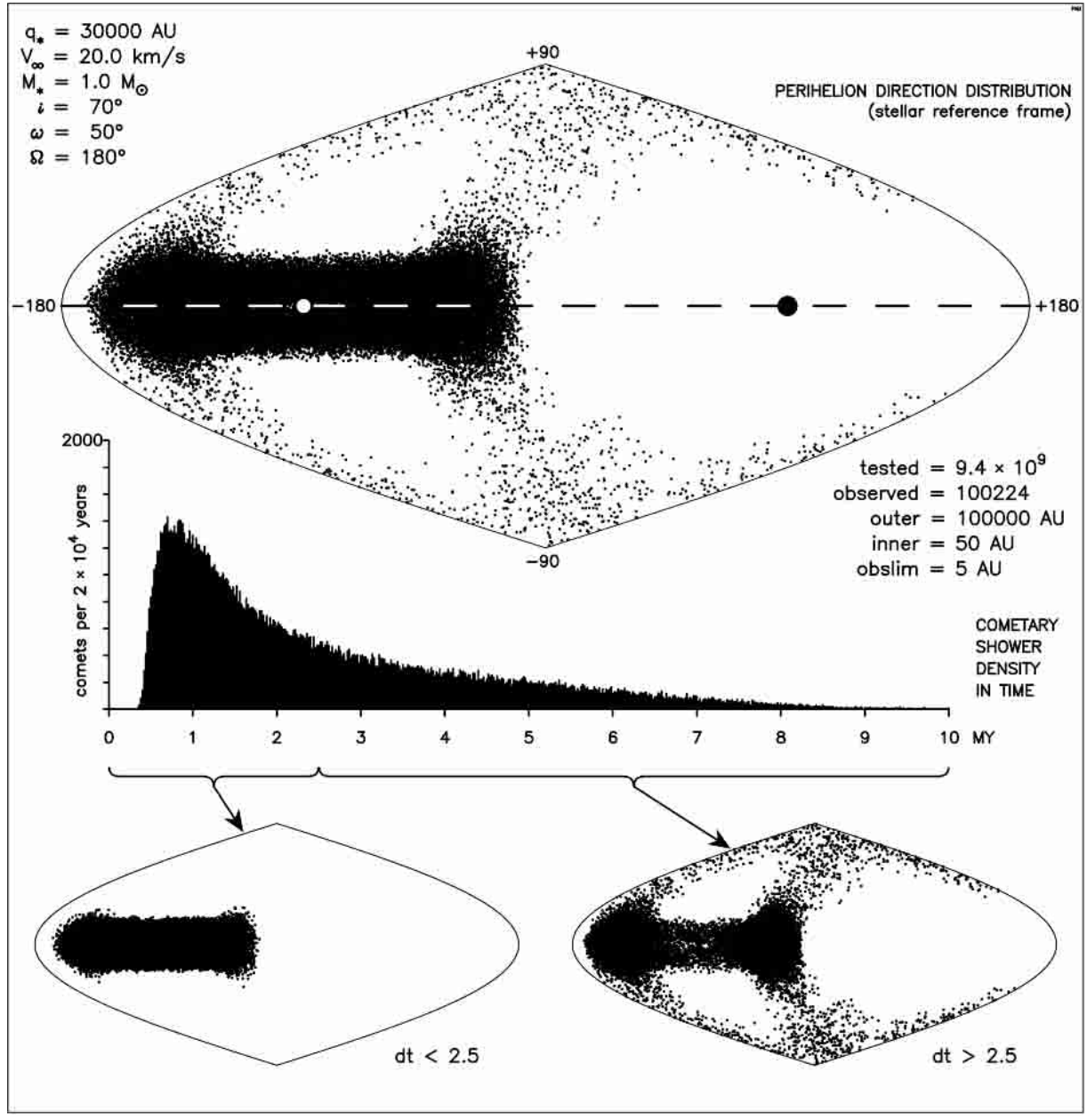

Fig. 8. The results of the simulation for relatively close stellar passage $\left(q_{*}=30000 \mathrm{AU}\right)$. Star heliocentric orbit plane projection is marked here with the dashed line, its perihelion with the full circle and its anti-perihelion with the open one. The cometary shower starts approx. 0.5 Myr after the star passage and appears as the steep increase of the observable flux. Below the arrival time distribution plot one can find two separated distributions for first $2.5 \mathrm{Myr}$ after the passage and the rest of the simulated observable sample. The left of these plots consists of more than $62 \%$ of all points presented in the upper plot. directional distribution to be depleted in equatorial and polar regions in the galactic frame. The other asymmetries needs different explanation. Matese et al. (1999) showed that a statistically significant grouping of the long-period comet aphelia directions along some great circle on the celestial sphere can be observed. Taking into account the Weissman (1996) conclusion that close stellar passage cannot be the source of such a grouping they propose a massive perturber to exist in the Oort cloud. However, Dybczyński (2002a,b) showed that Weissman's conclusions are applicable only for a very peculiar case and in general, stellar passages (both close and distant) produce highly anisotropic populations of the observable longperiod comets. Examples of such asymmetries are shown below. Matese \& Lissauer (2002) used a semi-analytic approach to study the combined effect of galactic and weak stellar perturbations on the Oort cloud comets. We will discuss this paper in more detail in the second paper of this series. We only mention here that they observe some aphelion distribution asymmetries in galactic latitude (similar to previous results) and longitude, especially for comets arriving in the Sun vicinity soon after the stellar passage. The last conclusion is in good agreement with our results presented below.

Figure 7 shows the distribution of perihelion points of the observable comets resulting from the stellar passage at $60000 \mathrm{AU}$ from the Sun. In the left, upper corner all parameters of the perturbing star are presented. Angular elements (referred to the galactic disk plane) are included here only for the sake of comparison with future results accounting for galactic perturbations. The presented distribution is plotted in the "stellar" reference frame so they are obsolete here. Below the equal-area projection of the celestial sphere we show the time dependence of the observable cometary flux by means of the histogram showing the number of comets per 20000 year time-interval. Perihelion direction points (and corresponding histogram bars) for comets arriving in the vicinity of the Sun during the first two million years after the star passage are marked in black, while the rest is in gray. The dashed line is the star heliocentric orbit projection onto the celestial sphere with its perihelion marked by the filled circle and its anti-perihelion at the position of the open circle. As it is easy to observe, the black points are more concentrated in the vicinity of the stellar anti-perihelion than the gray ones. Some parameters of the Monte Carlo simulations are also presented: from the cloud of $1.0 \times 10^{10}$ bodies we obtained 6146 observable comets; the probability of being observed is estimated here to be equal $(6.46 \pm 0.61) \times 10^{-7}$.

The example presented in Fig. 7 was calculated for DQT model of the cloud and for rather weak (and therefore more probable) stellar perturbation. The maximum observable cometary flux is obtained here approximately two million years after the star passage. If scaled for the cloud population of $10^{12}$ 


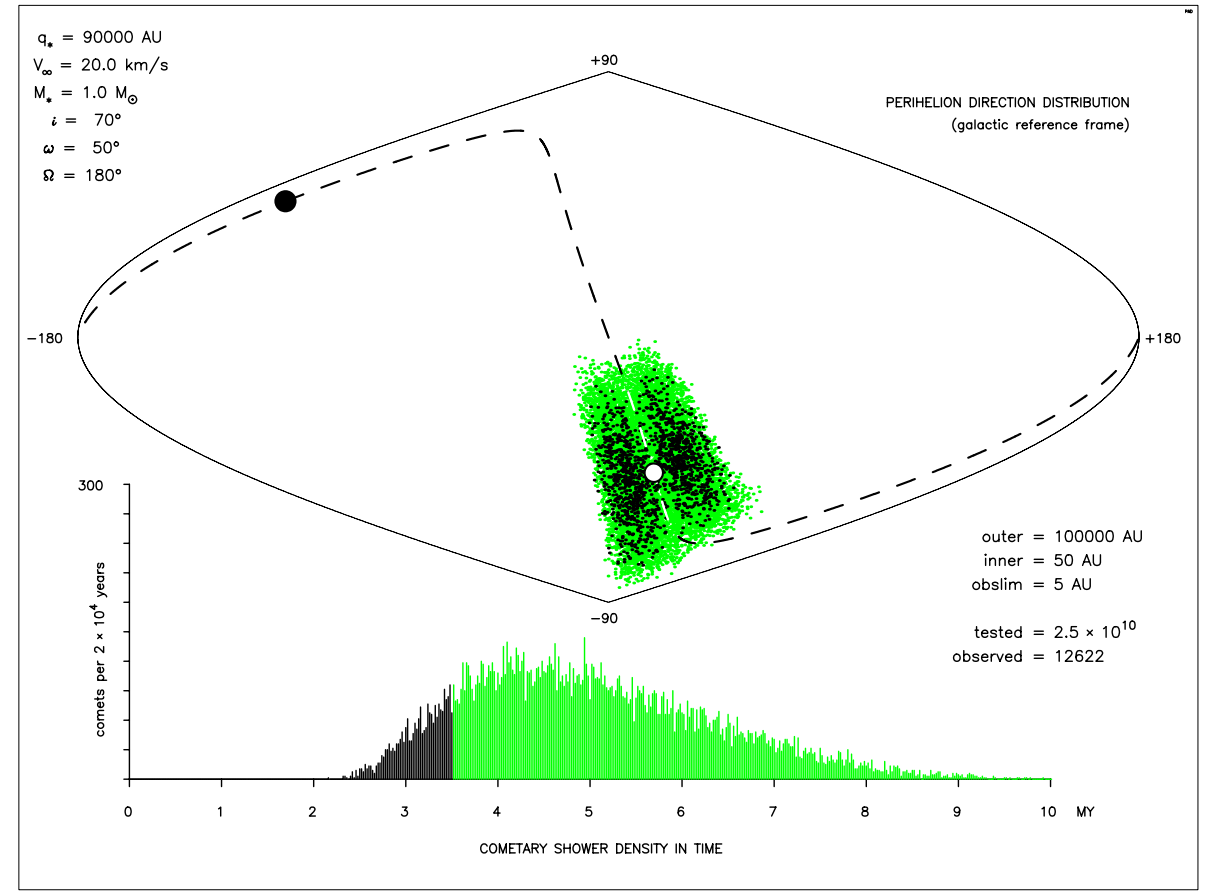

Fig. 9. The results of the simulation for the distant $\left(q_{*}=90000 \mathrm{AU}\right)$ stellar passage and the DLDW model of the cloud. All perihelion directions are highly concentrated on the celestial sphere, presented here in the galactic reference frame. The dashed line represents the projection of the star heliocentric orbit plane with perihelion and antiperihelion directions marked with full and open circle respectively. Comets arriving in the solar vicinity during the first 3.5 Myr after the stellar passage are marked in black while the rest is plotted in gray. To obtain a valuable statistics it was necessary to test the cloud population as large as $2.5 \times 10^{10}$ bodies. it will give one comet per 3 years during the peak. The same simulation performed for the DLDW model gives the same distribution of perihelion point directions. However, because the probability of being observed is significantly greater for this model, equal to $(2.71 \pm 0.26) \times 10^{-6}$, the observable cometary flux is also over three times greater, approximately one comet a year when scaled to the cloud population of $10^{12}$. Assuming that reasonable cometary data were obtained only during the last 200 years, the simulated star passage at $60000 \mathrm{AU}$ from the Sun, if it occurred two million years ago, would be the source of 70-200 comets among the observed long-period sample (assumming that we can observe all comets that pass within $5 \mathrm{AU}$ of the Sun).

The results for the closer stellar passage $\left(q_{\star}=30000 \mathrm{AU}\right)$ are presented in Fig. 8. It shows the results of a Monte Carlo simulation for the DLDW model of the cloud. The perturbations from such a passage were applied to $9.4 \times 10^{9}$ comets, obtaining 100224 observable ones. The estimated probability of being observed in this case is equal to $(1.08 \pm 0.03) \times 10^{-5}$. Because the directional distribution is more complicated here, at the bottom of Fig. 8 we present two additional plots for two separated time-intervals to emphasize that during the first 2.5 Myrs the concentration of the perihelion directions around the star anti-perihelion direction is very high and similar to the more distant passage case. The left, small plot consists of $62 \%$ of points from the upper sphere. But even for the whole 10 Myr interval, almost half of the celestial sphere is completely empty and the overall directional distribution orientation is aligned with the heliocentric star orbit. Note that the time-dependence histogram has different scale here: the maximum cometary flux reaches in this case 7 long-period comets per year when scaled to the cloud population of $10^{12}$.

This figure shows that contrary to the conclusions made by Weissman (1996) there is a strong signature of the stellar passage geometry in the population of observable comets produced by a close passage. The high concentration of perihelion directions may be observed especially for a distant stellar passage. The example of such a distribution, obtained for the DLDW model, is presented in Fig. 9. We introduce here the use of a galactic reference frame anticipating the presentation of the mixed stellar and galactic perturbation results, to be presented in paper two of this series. The probability of being observed is estimated here to be $(2.71 \pm 0.25) \times 10^{-6}$. An example of a very close stellar passage $\left(q_{\star}=10000 \mathrm{AU}\right)$ has been presented and discussed in detail in two recent papers (Dybczyński 2002a,b).

\section{Cometary shower characteristics}

There are three main features of the cometary shower induced by the close stellar passage near or through the Oort cloud: (i) time dependence of the induced observable comets influx, (ii) spatial distribution of the perihelia directions and (iii) semimajor axis distribution of comets observable during the shower. The first two features are clearly presented in Figs. 7-9 of the previous section. The third one may be seen in Figs. 10-12. All of them show the results of the simulated passage of one solar mass perturber at a velocity of $20 \mathrm{~km} \mathrm{~s}^{-1}$. The proximity distance of the perturbing star varies from $30000 \mathrm{AU}$ to 90000 $\mathrm{AU}$ as specified in the figure legends. We present a pair of histograms in each of these figures. Printed in light-gray is the semimajor axis distribution of observable shower comets for the DLDW model of the cloud and on top of this, marked in dark gray, the distribution for the DQT model is presented. In each case both simulations were performed for the same cloud population, therefore Figs. 10-12 show also the comparison of the model efficiency in producing observable comets with a single stellar passage. 


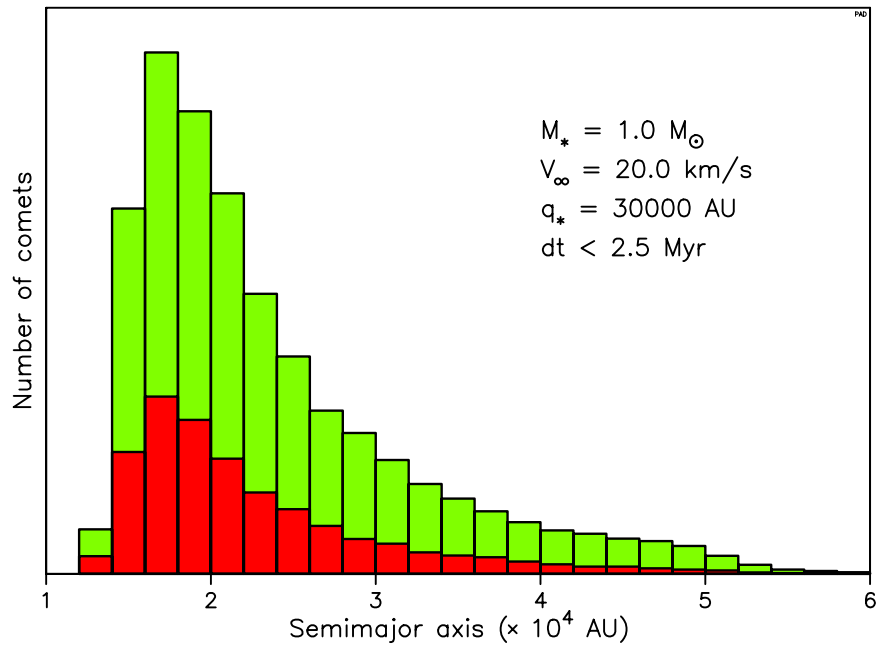

Fig. 10. Semimajor axis distribution for $q_{\star}=30000 \mathrm{AU}$.

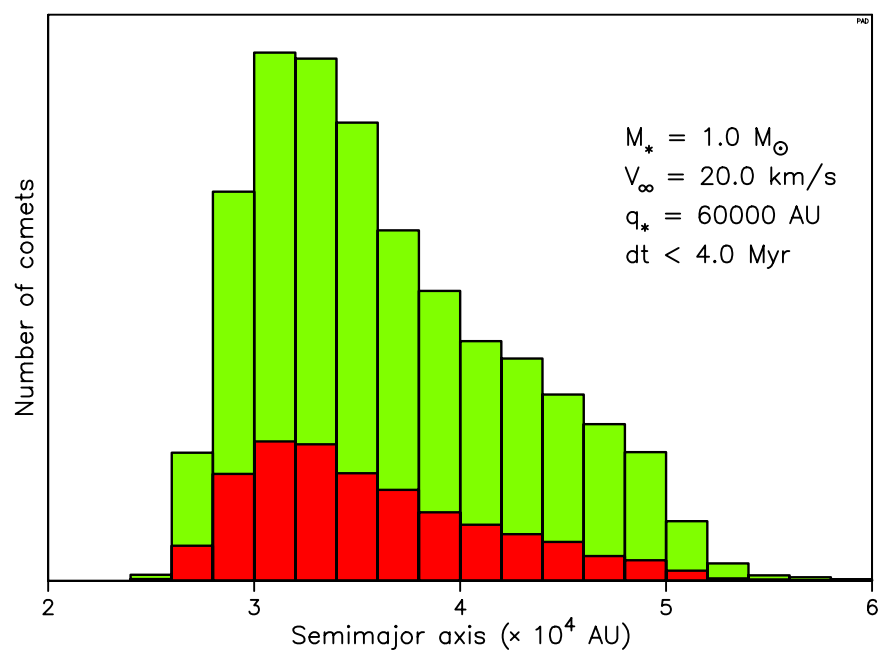

Fig. 11. Semimajor axis distribution for $q_{\star}=60000 \mathrm{AU}$.

Table 1. Cometary shower characteristics for the different proximity distances. The presented maximum cometary flux is in each case scaled to the cloud population of $10^{12}$ bodies. In all cases $\gamma=0.05$.

\begin{tabular}{ccccc}
\hline \hline & \multicolumn{2}{c}{ Main shower } & \multicolumn{2}{c}{ max. flux (per century) } \\
\cline { 2 - 5 }$d_{\star}$ & start & end & DLDW & DQT \\
\hline $30000 \mathrm{AU}$ & $0.5 \mathrm{Myr}$ & $2.5 \mathrm{Myr}$ & 700 & 250 \\
$60000 \mathrm{AU}$ & $1.5 \mathrm{Myr}$ & $4 \mathrm{Myr}$ & 100 & 35 \\
$90000 \mathrm{AU}$ & $3.3 \mathrm{Myr}$ & $6 \mathrm{Myr}$ & 30 & 5 \\
\hline
\end{tabular}

The sample of observable comets constituting these histograms is limited in each case to the time interval covering the neighbourhood of the shower flux peak. For the distance of $30000 \mathrm{AU}$ we decided to include all comets that arrive at perihelion no later than 2.5 Myr after the stellar passage. In Figs. 11 and 12 the corresponding time limit is adopted to be equal to 4 and $6 \mathrm{Myr}$ respectively. This allow us to concentrate on the shower itself, not on the background of weakly perturbed comets that arrive at perihelion later.

Table 1 summarizes the shower characteristics. The cometary fluxes estimated here are the results of a single stellar

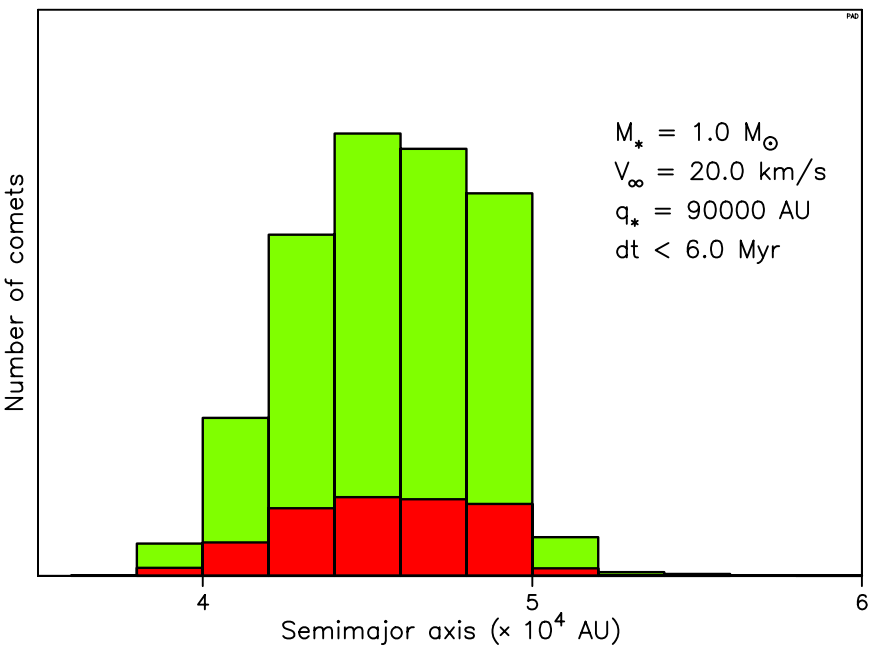

Fig. 12. Semimajor axis distribution for $q_{\star}=90000 \mathrm{AU}$.

passage in the absence of any other source of perturbation. The main shower starting and ending moments were chosen here in a rather arbitrary manner: we used time intervals when the cometary flux is greater than some $30 \%$ of the maximum value.

\section{Conclusions}

In this paper we presented the detailed analysis of the result of single stellar passage near or through the Oort cometary cloud in the absence of any other perturbing forces. We showed that the population of the observable comets induced by such a passage shows strong asymmetry in the perihelion direction distribution. We demonstrated the time-dependence of this concentrations as well as the time variation of the observable comet influx. We also presented the dependence of the various endstate probabilities on the stellar perturbation strength parameter as well as on the proximity distance. The above results are obtained separately for two different cometary cloud models. The dependence of the main features of the cometary shower on the cloud model is also shown.

Among the detailed conclusions we mention that:

1. The flattened inner part of the DLDW model does not appear in our results. We performed several additional simulations with different geometrical orientations of the stellar path and obtained no dependence on it. The reason may be found in Fig. 10. Even for such a close passage we do not observe comets with semimajor axes smaller than $10000 \mathrm{AU}$ and outside this threshold value the DLDW model is spherically symmetric.

2. The most important differences between DQT and DLDW models are the end-state probabilities. In almost all cases the DLDW model produces 3-4 times more observable comets. For the distant passage the relative efficiency of this model is even greater, as can be found in Fig. 12. The reason for this effect is the more populated outer part of the cloud in the DLDW model, as illustrated in Fig. 3. The directional distributions of the observable comet perihelions are practically the same for both models. 
3. For both models the probability of "being lost" is approximately 100 times greater then the probability of "being observed". This means that during each stellar passage at any proximity distance two orders more comets were lost than entered the inner planetary system.

4. The influx of observable comets induced by a stellar passage is generally very low. Even for a close passage $\left(q_{\star}=30000 \mathrm{AU}\right)$ and DLDW model we obtained approximately 7 comets per year during the maximum shower activity.

5. When searching for stellar passage fingerprints one has to concentrate on very recent (last few Myrs) events. Because of the high level of perihelion direction concentration on the celestial sphere it seems to be possible to recognize such patterns even for the combined output of several passages.

The results presented in this paper are important because they constitute the basis for extended studies when stellar and galactic perturbations act simultaneously. In the next paper of this series we will present the results of such extended simulations.

Acknowledgements. I would like to thank Luke Dones, the referee of this paper, for his valuable remarks and suggestions. This work was partially supported by the KBN Grant No. 2P03D00722

\section{References}

Bailey, M. E., Clube, S. V. M., \& Napier, W. M. 1990, The Origin of Comets (Pergamon Press)

Biermann, L., Huebner, W. F., \& Lüst, R. 1983, Proc. Natl. Acad. Sci. USA, 80, 5151

Bogart, R. S., \& Noerdlinger, P. D. 1982, AJ, 87, 911

Breiter, S., Dybczyński, P. A., \& Elipe, A. 1996, A\&A, 315, 618

Brunini, A. 1993, A\&A, 273, 684

Carrington, R. C. 1861, MRAS, XXIX, 355

Delsemme, A. H. 1987, A\&A, 187, 913

Dones, L., Levison, H., Duncan, M., \& Weissman, P. 2000a, Formation of the Oort Cloud, pers. comm.

Dones, L., Levison, H., Duncan, M., \& Weissman, P. 2000b, in AAS/Division of Planetary Sciences Meet., vol. 32, 3602

Duncan, M., Quinn, T., \& Tremaine, S. 1987, AJ, 94, 1330

Dybczyński, P. A. 1990, in Nordic-Baltic Astronomy Meet., ed. C.-I. Lagerkvist, D. Kiselman, \& M. Lindgren (Uppsala: Uppsala universitet), 89

Dybczyński, P. A. 1994, Celestial Mechanics and Dynamical Astronomy, 58, 139

Dybczyński, P. A. 2002a, in Proc. of IAU Colloq. 186: Cometary Science after Halle-Bopp, to be published

Dybczyński, P. A. 2002b, A\&A, 383, 1049

Dybczyński, P. A., \& Prȩtka, H. 1996, Earth Moon and Planets, 72, 13

Fernández, J. A. 1980, Icarus, 42, 406

Fernández, J. A. 1982, AJ, 87, 1318

Fernández, J. A., \& Ip, W.-H. 1987, Icarus, 71, 46
García-Sánchez, J., Preston, R. A., Jones, D. L., et al. 1999, AJ, 117, 1042

García-Sánchez, J., Weissman, P. R., Preston, R. A., et al. 2001, A\&A, 379,634

Hasegawa, I. 1976, PASJ, 28, 259

Heisler, J. 1990, Icarus, 88, 104

Heisler, J., Tremaine, S., \& Alcock, C. 1987, Icarus, 70, 269

Heisler, J., Tremaine, S., Weissman, P., \& Greenberg, R. 1991, in Lunar and Planetary Science Conf., vol. 22, 553

Heisler, J., \& Tremaine, S. D. 1986, Icarus, 65, 13

Hills, J. G. 1981, AJ, 86, 1730

Hurnik, H. 1964, in Prace Wydziału Matematyki, Fizyki i Chemii, Seria Astronomia No. 1 (Poznań: Uniwersytet im. A. Mickiewicza), 1

Hut, P., Alvarez, W., Elder, W. P., et al. 1987, Nature, 329, 118

Joss, P. C. 1973, Icarus, 19, 147

Khana, M., \& Sharma, S. D. 1983, PASJ, 35, 559

Lüst, R. 1984, A\&A, 141, 94

Maciejewski, A. J., \& Prętka, H. 1998, A\&A, 336, 1065

Matese, J. J., \& Lissauer, J. J. 2002, Icarus, 157, 228

Matese, J. J., \& Whitman, P. G. 1989, Icarus, 82, 389

Matese, J. J., \& Whitman, P. G. 1992, Celestial Mechanics and Dynamical Astronomy, 54, 13

Matese, J. J., Whitman, P. G., \& Whitmire, D. P. 1998, Celestial Mechanics and Dynamical Astronomy, 69, 77

Matese, J. J., Whitman, P. G., \& Whitmire, D. P. 1999, Icarus, 141, 354

Matese, J. J., \& Whitmire, D. 1996, ApJ, 472, L41

Oja, H. 1975, A\&A, 43, 317

Oort, J. H. 1950, Bull. Astron. Inst. Nether., 11, 91

Öpik, E. 1932, Proc. Amer. Acad. of Arts and Sci., 67, 169

Remy, F., \& Mignard, F. 1985, Icarus, 63, 1

Rickman, H. 1976, Bull. Astr. Inst. Czechoslov., 27, 92

Rickman, H., \& Froeschle, C. 1988, Celestial Mechanics, 43, 243

Scholl, H., Cazenave, A., \& Brahic, A. 1982, A\&A, 112, 157

Tyror, J. G. 1957, MNRAS, 117, 370

Weissman, P., \& Hut, P. 1986, in Lunar and Planetary Science Conference, vol. 17, 935

Weissman, P. R. 1977, in Comets, asteroids, Meteorites - interrelations, evolution and origins, ed. A. H. Delsemme (Toledo, Ohio, USA: Univ. of Toledo), 87

Weissman, P. R. 1979, in Dynamics of the Solar System, ed. R. L. Duncombe (Dordrecht, Holland: D. Reidel Publ. Co.), IAU Symp., 81, 277

Weissman, P. R. 1980, Nature, 288, 242

Weissman, P. R. 1982, in Comet Discoveries, Statistics, and Observational Selection, IAU Coll., 61, 637

Weissman, P. R. 1984, Nature, 312, 380

Weissman, P. R. 1996, Earth, Moon and Planets, 72, 25

Yabushita, S. 1979a, in Dynamics of the Solar System, ed. R. L. Duncombe, IAU (Dordrecht-Holland: D. Reidel Publ. Co.), 283

Yabushita, S. 1979b, MNRAS, 189, 45

Yabushita, S., Hasegawa, I., \& Kobayashi, K. 1982, MNRAS, 200, 661 